\title{
DESARROLLO DE UN PROCEDIMIENTO ESTRUCTURADO DE OBSERVACIÓN CLIINICA DE LA MODULACIÓN TÁCTIL Y DESEMPEÑO DE DOS MUESTRAS CLÍNICAS DE NIÑOS CON Y SIN DEFENSIVIDAD TÁCTIL
}

\author{
DEVELOPMENT OF A STRUCTURED PROCEDURE OF CLINICAL OBSERVATIONS \\ OF THE SENSORY MODULATION AND THE PERFORMANCE OF TWO CLINICAL \\ SAMPLES OF CHILDREN WITH AND WITHOUT TACTILE DEFENSIVENESS
}

\section{Daniel Esteban Calderón Larraín}

\begin{abstract}
RESUMEN
La defensividad táctil es una de las disfunciones de integración sensorial que fueron tempranamente descritas por Jean Ayres (Lane, 2020). Sin embargo, para su diagnóstico se carece de instrumentos de evaluación basados en la observación del desempeño del niño, y en cambio, se suele utilizar casi exclusivamente el reporte de los padres por medio de cuestionarios sensoriales.

Basado en diversos fundamentos teóricos se diseñó un procedimiento estructurado de observación clínica de la modulación táctil, compuesto por dos juegos con exposición a estímulos táctiles, el cual fue aplicado en contexto de práctica clínica privada, registrándose sus observaciones textualmente en fichas clínicas. El objetivo de esta investigación es describir los signos de defensividad táctil que pueden ser detectados con este procedimiento.

Por medio de una pauta de análisis de 29 ítems de respuesta dicotómica, se examinaron retrospectivamente 83 fichas, de una población clínica de niños con y sin defensividad táctil, de 2 a 11 años de edad. Se comparó los resultados de una muestra de niños con defensividad táctil con otra de niños sin defensividad táctil, por medio de estadística descriptiva y pruebas no paramétricas.

El desempeño de niños con defensividad táctil estadísticamente difiere de manera significativa del de niños sin defensividad táctil en el procedimiento de observación estudiado. Además, este instrumento puede contribuir con el proceso diagnóstico de defensividad táctil, por medio de la contabilización de indicadores para sugerir el diagnóstico, o de la identificación específica de algunos de ellos que serían más relevantes.
\end{abstract}

\section{PALABRAS CLAVE}

Defensividad táctil, trastornos de modulación, integración sensorial

1 Magíster en Estrategias de Intervención en Salud Mental Infantil, Universidad del Desarrollo. Licenciado en Ciencias de la Ocupación Humana, Universidad de Chile. Terapeuta Ocupacional, Universidad de Chile. Centro Huella Chicureo. Académico Universidad de Los Andes Camino El Alba 2 Parcela 38 Lote 5, Colina. danielcalderon.to@gmail.com dcalderon@huellachicureo.cl.988880284 


\begin{abstract}
Tactile defensiveness is one of the first sensory integration dysfunctions described by Jean Ayres. However, for the diagnosis, evaluation procedures based on the observation of the child's performance are lacking, and instead, parents' reports are usually used almost exclusively.

Based on several theoretical foundations, a structured procedure of clinical observations of tactile modulation was designed, consisting of two games that expose children to tactile stimuli. The observation was applied in a private clinic, using recording used for the purpose of documentation in clinical records. The objective of this research is to describe the tactile defensiveness signs that may be detected with this procedure.

Through a guideline with 29 items of dichotomous response, 83 clinical records were retrospectively examined, from a clinical population of children with and without tactile defensiveness, aged 2 to 11 years. The results of a sample of children with tactile defensiveness were compared with another one of children without tactile defensiveness, through descriptive statistics and non-parametric tests. The performance of children with tactile defensiveness are significantly different from children without tactile defensiveness. In addition, this instrument can contribute to the diagnostic process of tactile defensiveness, through the analysis of indicators that suggest the diagnosis, or the specific identification of relevant signs of tactile defensiveness.
\end{abstract}

\title{
KEYWORDS
}

Tactile defensiveness, sensory modulation disorders, sensory integration

Recepcionado: 03/09/2020

Aceptado: 28/11/2020

\section{INTRODUCCIÓN}

Las diversas disfunciones de integración sensorial pueden ser agrupadas en dos amplias categorías: trastornos de discriminación y trastornos de modulación (Bundy \& Lane, 2020). Mientras que los trastornos de discriminación impactan principalmente el desempeño motor y específicamente la praxis, los trastornos de modulación se asocian con dificultades en la regulación de la conducta y emociones, vinculado con el nivel de alerta (Bundy \& Murray, 2002). Miller, Coll y Schoen (2007) definieron a los trastornos de modulación como las dificultades para regular la intensidad y la naturaleza de las respuestas a los estímulos sensoriales, causando problemas en la participación ocupacional, y éstos están referidos a alguno de los siguientes subgrupos: individuos que presentan una demanda inusualmente alta de estímulos sensoriales (búsqueda de sensaciones), personas que ignoran o no registran ciertos estímulos sensoriales (hiporresponsividad sensorial), o individuos que responden intensamente, con mayor rapidez o por más tiempo a las sensaciones (hiperresponsividad sensorial) (Miller, Anzalone, Lane, Cermak \& Osten, 2007).
Las respuestas exageradas a los estímulos sensoriales, que ocurren en la hiperresponsividad sensorial, se asocian con problemas funcionales en la autorregulación, con el temperamento "difícil", con dificultades en la interacción social, y con disconfort emocional (Bates, Bennett Freeland \& Lounsbury, 1979; Rothbart, Ahadi, Hershey \& Fisher, 2001), con problemas socioemocionales del dominio internalizante (ansiedad, depresión, aislamiento), externalizante (agresión y desafío, hiperactividad e impulsividad) y desregulación (emocionalidad negativa, dificultades en sueño y alimentación) (BenSasson, Carter \& Briggs-Gowan, 2009), y también con ansiedad en niños con trastorno por déficit de atención con hiperactividad, ánimo negativo en bebés de un mes de vida y en preescolares con trastorno del espectro autista (Dunn, Little, Dean, Robertson \& Evans, 2016).

La defensividad táctil se considera un tipo de hiperresponsividad sensorial relacionada específica y principalmente (y no exclusivamente) con los estímulos táctiles. Ha sido una de las primeras disfunciones de integración sensorial descritas por Jean Ayres (desde 1964), y una de las más discutidas (Lane, 2020). Puede afectar 
la participación en las actividades de la vida diaria y el juego del niño desde temprana edad y de manera generalizada (Mailloux \& Burke, 2008). Según descripción de Royeen y Lane (1991), la defensividad táctil hace referencia a conductas observables de aversión a ciertos estímulos táctiles que para la mayoría de las personas no son nocivos, por lo cual un toque ligero puede provocar una conducta de protección, de escape y respuestas emocionales intensas (Lane, 2020). En síntesis, la defensividad táctil corresponde a una disfunción caracterizada por un patrón regular de respuestas exageradas y aversivas al estímulo táctil, que funcionalmente afectan la participación ocupacional, la regulación emocional y la conducta.

A pesar de que la defensividad táctil ha sido ampliamente descrita (Royeen \& Lane, 1991), sus procedimientos de detección y diagnóstico no han sido suficientemente sofisticados (Bundy, 2002), se basan especialmente en cuestionarios sensoriales para padres y cuidadores (Ben-Sasson, et al., 2009; Eeles, et al., 2013), observaciones no estructuradas (Blanche \& Reinoso, 2008), o habilidades de razonamiento clínico (Royeen \& Lane, 1991). Mientras, para los trastornos de praxis se utilizan procedimientos de evaluación estandarizados como el Sensory Integration and Praxis Tests (SIPT) (Bundy, 2002; Mulligan, 202O), otros instrumentos estandarizados del desempeño motor que pueden ser interpretados desde la perspectiva de integración sensorial (Bundy, 202O; Eeles, et al., 2013), observaciones clínicas estructuradas relacionadas con el procesamiento vestibular y propioceptivo (Blanche \& Reinoso, 2008; Blanche, 2010; Blanche, Bodison, Chang, \& Reinoso, 2012; Blanche, Reinoso \& Blanche Kiefer, 2014; Blanche, Reinoso \& Blanche Kiefer, 202O), e incluso existen normas estandarizadas de discriminación táctil (en SIPT y su precursor, SCSIT, Southern California Sensory Integration Test) (Ayres, 1980; Blanche \& Reinoso, 2008). Esto ha puesto en rezago la evidencia referida a los trastornos de modulación: en los diversos estudios de análisis de factores raramente se han incluido variables de trastornos de modulación (Mailloux, et al, 2011), y Ayres sólo lo hizo para la defensividad táctil (Bundy \& Murray, 2002).

La utilización de cuestionarios sensoriales para cuidadores (tales como Sensory Processing Measure [SPM], o Sensory Profile 2) tiene la ventaja de proveer información referida a la vida cotidiana del niño, considerando que ciertas conductas podrían ocurrir en contextos específicos y no en un ambiente clínico, sin embargo las respuestas dependen del criterio variable de los cuidadores (Ben-Sasson, et al., 2009), y aun cuando existen traducciones oficiales en español (Dunn, 2014), éstas deberían ser adaptadas localmente, para que los resultados sean válidos y confiables.

En el contexto de la validación del trastorno de modulación como categoría diagnóstica independiente, se desarrollaron procedimientos experimentales para la identificación de la hiperresponsividad sensorial por medio de protocolos e indicadores como la conductividad electrodérmica (Miller, Reisman, Mclntosh \& Simon, 2001), pero su costo es elevado y su implementación es difícil para la práctica clínica.

Mientras se hace necesario el desarrollo de una evaluación de la modulación sensorial, de "desempeño" (aplicada directamente al individuo) (Schoen, Miller \& Green, 2008), y según criterio profesional (Schoen, Miller \& Sullivan, 2014), sólo son conocidos procedimientos no estandarizados ni estructurados de observación, y compilados de conductas (Blanche \& Reinoso, 2008; Blanche, 2010; Bundy, 2002).

En el manual de SCSIT (y también en SIPT), por ejemplo, se sugieren observaciones clínicas durante la aplicación de los tests de discriminación táctil que permitirían detectar la defensividad táctil, como eventuales respuestas defensivas que ocurren cuando el niño no observa donde lo están tocando, o cuando el niño verbaliza sentir cosquillas o que se pincha, o reacciones más evidentes, emocionalmente intensas, agresivas o de huida (Ayres, 1980).

Más allá de tales observaciones incidentales, Royeen (1986) promovió refinar los procedimientos de evaluación a través de un instrumento estandarizado del desempeño de la persona; entonces desarrolló un cuestionario sensorial dirigido al niño acerca de situaciones cotidianas que involucran estímulos táctiles: Touch Inventory for Elementary School-Age Children (TIE) (Bundy, 2002).

Royeen y Lane (1991) describieron una serie de conductas de evitación al tacto, de respuestas aversivas al tacto no nocivo, y de respuestas afectivas atípicas al tacto no nocivo, las cuales deberían observarse en un patrón consistente (en intensidad o cantidad de reacciones negativas al tacto) (Lane, 2002). Además, propusieron procedimientos de observación basados en un juego 
táctil (con pintura para dedos, espuma de afeitar, burbujas) para observar respuestas defensivas.

Blanche (2010) entrega sugerencias para la observación de las respuestas del niño frente al input táctil, por ejemplo: tendencia para explorar usando sus manos, comodidad con distintas experiencias táctiles, conducta de huida o signos de ansiedad. Además, muestra la aplicación de diferentes estímulos táctiles.

Miller y Summers (2001) desarrollaron un listado de comportamientos en los trastornos de modulación, calificando según el grado de apoyo necesario por el evaluador para continuar con la evaluación.

De manera paralela a lo presentado en este artículo, Lista y Blanche (2020) desarrollaron la Evaluación del Procesamiento Táctil (EPT), cuya versión final consiste en 13 ítems agrupados según estímulos aplicados en manos o brazos, cara o cabeza, y pies, con una escala única de calificación de 7 puntos para todos los ítems, de acuerdo al nivel de respuesta a cada uno.

El esfuerzo más sistemático para desarrollar un instrumento de evaluación basado en el desempeño del individuo, y con criterio profesional, acerca de los trastornos de modulación ha sido la serie de investigaciones que inicialmente desarrollaron el Sensory Over-Responsivity Scales (SensOR) (Schoen, et al., 2008), derivando posteriormente en Sensory Processing Scale Assessment (Schoen, et al, 2014), y finalmente en Sensory Processing 3-Dimensions Scale (Mulligan, Schoen, Miller, Valdez \& Magalhaes, 2019). Ninguno de estos tres instrumentos está aún disponible comercialmente. En lo referido a esta investigación, estos instrumentos proponen diversos ítems y tareas para detectar defensividad táctil (y otros signos de trastornos de modulación).

Con el propósito de complementar el reporte de los cuidadores (por medio de entrevistas o cuestionarios) y las observaciones clínicas no estructuradas, con una observación del desempeño, en 2014 se diseñó y estructuró un procedimiento de evaluación de la modulación táctil (Calderón, 2016), el cual consiste en dos juegos con exposición a materiales táctiles y observaciones incidentales, basándose en diversos fundamentos teóricos provenientes de la literatura:

1. En primer lugar, está estructurado en un contexto de juego, buscando involucrar activamente al niño en la actividad, asumiendo el mismo terapeuta una actitud de juego (Mailloux \& Burke, 2008), y evitando un daño en el vínculo terapéutico tras la exposición a un material potencialmente estresante (los estímulos táctiles).

2. Para controlar algunas variables relacionadas con la función modulatoria de la propiocepción, se sugiere que el niño no haya realizado previamente actividad física intensa (Blanche \& Schaaf, 2001), ya que los signos de defensividad táctil podrían no observarse.

3. Se otorga una exposición reiterada a los estímulos y se realizan dos juegos de manera continua, para aumentar la probabilidad de observar la respuesta defensiva, ya que ésta tiende a ser acumulativa o a empeorar luego de la exposición repetitiva (Bundy, 2002), o bien, aumenta paulatinamente en vez de aparecer de súbito (Ben-Sasson, et al., 2009). Por otra parte, en la población típica puede esperarse una reacción inicial alta con algún estímulo y luego habituación, mientras que en niños con defensividad táctil pueden presentar varios peak de respuesta y no presentan habituación ante la reiteración (Miller, et al., 2001).

4. El juego es estructurado por el evaluador y los estímulos son otorgados de manera inesperada. Las respuestas defensivas se observan mayormente frente al tacto inesperado (Ben-Sasson, et al., 2009; Royeen, 1985): aún los niños con defensividad táctil tienden a tolerar mejor el estímulo táctil autoaplicado, en lugar del recibido pasivamente o de aquél que el niño no puede ver (Parham \& Mailloux, 2005). El contexto de juego facilita la percepción "casual" de la interacción con el material táctil, evitando dañar el compromiso del niño con la actividad. La realización de una observación estructurada por el evaluador, en vez de una observación de la conducta del juego libre (o sea, una observación no estructurada) (Blanche \& Reinoso, 2008), permite la entrega de estímulos específicos y de una forma particular por parte del terapeuta, disminuyendo el control del niño y permitiendo observar las conductas características.

5. La elección de los materiales del procedimiento de evaluación está basada en diversos estímulos mencionados recurrentemente en la literatura para la 
exploración de las reacciones al tacto, por ejemplo: espuma de afeitar (Royeen \& Lane, 1991; Blanche, 2010), sémola como material particulado similar a la arena (Bundy, 2002; Royeen \& Lane, 1991), semillas (Windsor, Roley, \& Szklut, 2001), pinceles y soplo de aire emulando materiales similares de SensOR (Schoen, et al., 2008).

Este procedimiento de evaluación de la modulación táctil fue aplicado en contexto de consulta clínica privada entre 2014 y 2016, en la comuna de Colina (Chile), y sus observaciones fueron registradas textualmente en las fichas clínicas.

El propósito de esta investigación es describir signos conductuales del procedimiento estructurado de observación clínica de la modulación táctil descrito, con el fin de proponer un nuevo instrumento de evaluación basado en el desempeño del individuo, que contribuya a la detección y diagnóstico de la defensividad táctil, complementando la información proveniente del reporte de los cuidadores y de las observaciones clínicas no estructuradas.

\section{MÉTOdo}

\section{Participantes}

Entre 2014 y 2016 el procedimiento estructurado de observación clínica de la modulación táctil fue aplicado en contexto de consulta clínica privada en la comuna de Colina, por parte de un profesional entrenado y certificado en integración sensorial, registrándose tales observaciones textualmente en las fichas clínicas.
De un universo de 126 evaluaciones efectuadas entre septiembre de 2014 y septiembre de 2016, se analizaron retrospectivamente 83 fichas clínicas que presentaban el procedimiento de evaluación descrito, correspondientes a niños y niñas cuyas edades al momento de la evaluación eran desde los 2 años 6 meses hasta los 11 años 8 meses (promedio = 5 años 4 meses; desviación estándar = 2 años 1 mes), y que fueron referidos a servicios de terapia ocupacional por dificultades funcionales en la regulación de emociones o conducta, por problemas en motricidad, o por sospecha de dificultades en el desarrollo, presentando diagnóstico médico o no. Los participantes provenían de familias pertenecientes al nivel socioeconómico alto.

Los individuos fueron separados en dos grupos, basándose en un algoritmo que combinaba motivo de consulta, referencia espontánea a síntomas táctiles en el motivo de consulta (Lane, 2002; Royeen \& Lane, 1991), y resultados en cuestionarios sensoriales para cuidadores (ver Figura 1), generándose un grupo de niños con defensividad táctil (DT) (44 individuos), y otro sin defensividad táctil (NO DT) (39 individuos) (ver Tabla 1), independientemente de la presencia de otro diagnóstico asociado o no. Tales posibles comorbilidades son: trastorno del desarrollo de la coordinación y dispraxia, trastorno por déficit atencional con o sin hiperactividad, trastorno o retraso en el lenguaje, retraso en el desarrollo psicomotor, trastornos emocionales y adaptativos, trastorno del espectro autista, síndrome de Down, trastorno de la regulación, u otra disfunción de integración sensorial.

De acuerdo con la definición, en el algoritmo propuesto, los individuos del grupo DT debían presentar dificultades de regulación de las emociones y de la conducta, y además síntomas táctiles mencionados espontáneamente en el motivo de consulta o por medio de los

TABLA 1 DISTRIBUCIÓN DE LA MUESTRA FINAL POR GRUPOS DIAGNÓSTICOS

\begin{tabular}{lll}
\hline & NO DT & DT \\
\hline n Total (\%) & $39(47 \%)$ & $44(53 \%)$ \\
Niños (\%) & $33(85 \%)$ & $39(89 \%)$ \\
Niñas (\%) & $6(15 \%)$ & $5(11 \%)$ \\
Intervalo etario & 2 años $7 \mathrm{~m}-11$ años $6 \mathrm{~m}$ & 2 años $6 \mathrm{~m}-11$ años $8 \mathrm{~m}$ \\
Edad promedio & 5 años $8 \mathrm{~m}$ & 5 años $0 \mathrm{~m}$ \\
DS Edad & 2 años $3 \mathrm{~m}$ & 1 año $11 \mathrm{~m}$ \\
\hline
\end{tabular}


cuestionarios sensoriales para cuidadores. Por otro lado, los individuos del grupo NO DT presentaban dificultades funcionales distintas a problemas en la regulación de las emociones y la conducta, o no debían presentar síntomas táctiles en el motivo de consulta ni tampoco en los cuestionarios sensoriales aplicados. Esta información fue procesada informáticamente para evitar la inclusión en los grupos de manera tautológica.

Cabe mencionar que a 14 individuos de la muestra final no se les dio indicación de tratamiento, debido a un rendimiento dentro de los parámetros esperados en los demás tests aplicados (normativos o de referencia a criterio), o por el bajo impacto de las dificultades en su participación cotidiana. De todos ellos, 7 correspondieron al grupo NO DT (es decir, niños de probable desarrollo típico), y 7 al grupo DT (probablemente niños con bajo impacto de las dificultades en su participación cotidiana).

\section{FIGURA 1 ALFORITMO DE DECISIÓN PARA LA CONFORMACIÓN DE LOS GRUPOS DIAGNÓSTICOS.}

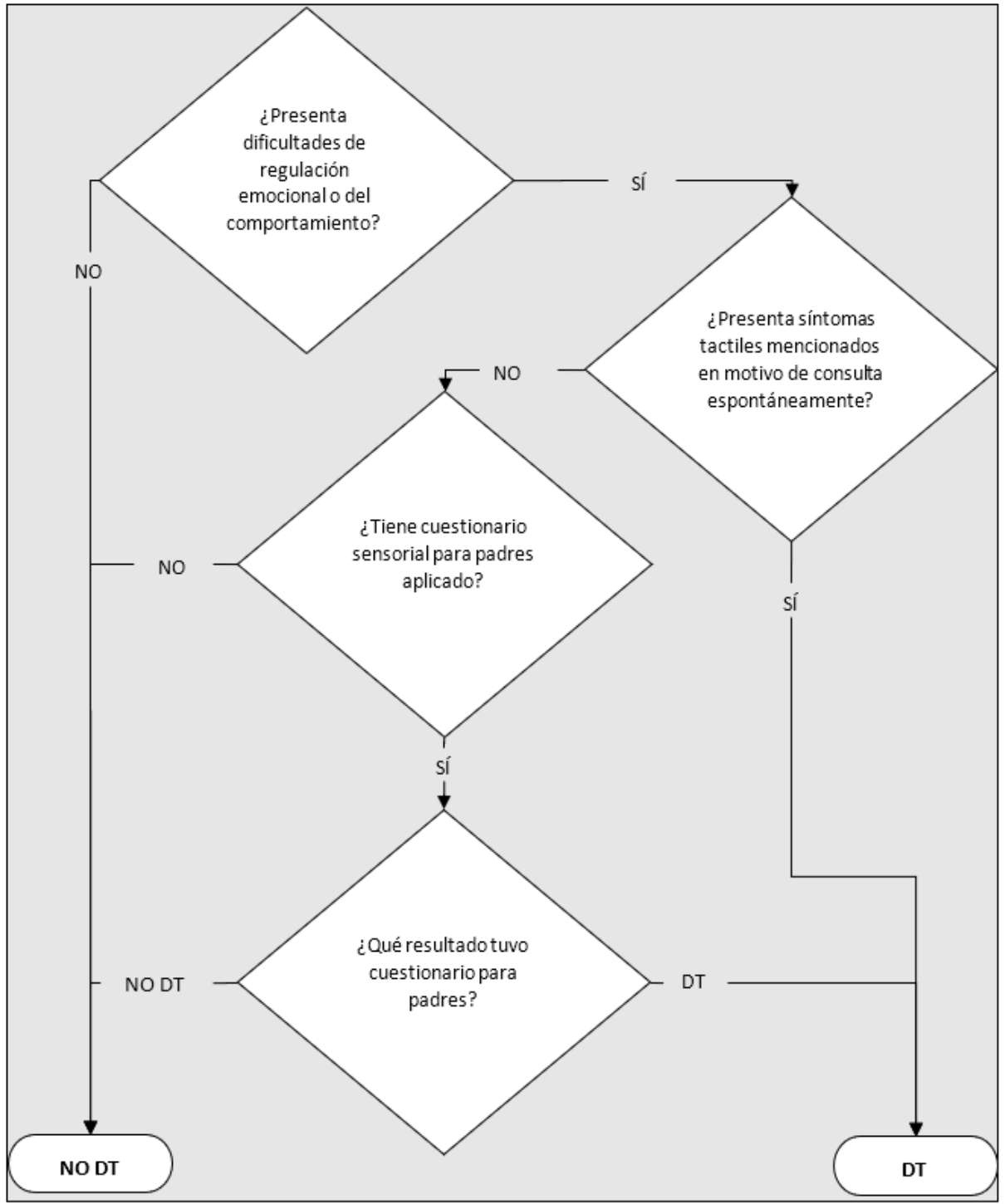




\section{Instrumento}

Para efectos de este estudio, se confeccionó una Pauta de Análisis del registro de las observaciones, compuesto por 29 ítems de respuesta dicotómica, de observaciones puntuales durante el procedimiento de observación clínica de la modulación táctil señalado, y agrupadas según los eventos de observación: juego con espuma, juego con sémola, reacción a pinceles y aire, observaciones generales y observaciones incidentales durante pruebas de discriminación táctil.

Los ítems son:

Juego con espuma

1. Muestra respuesta en miembros superiores.

2. Muestra respuesta en la cara o cuello.

3. El niño muestra una primera reacción elevada (grita, se molesta, expresa asco, o rechaza)

4. El niño expresa o muestra incomodidad en el contacto con la espuma (lo manifiesta directamente o lo expresa gestualmente)

5. El niño rechaza verbalmente continuar con el juego táctil, o intenta limpiarse el material.

6. El niño permanece en el juego táctil menos de 30 segundos.

7. El niño esparce la espuma de afeitar por un sector amplio de la colchoneta.

8. Respuesta inicial es reiterada y no disminuye (no se habitúa).

9. Presenta respuesta de freezing.

10. El niño aumenta su nivel de actividad significativamente, se desorganiza su conducta, se irrita emocionalmente o aumenta desatención.

Juego con sémola

1. Presenta respuesta al contacto no esperado con el material

2. Presenta una alta respuesta inicial.

3. El niño manifiesta incomodidad verbal o gestualmente con el contacto.

4. El niño rechaza involucrarse o continuar en el juego con el material.

5. El niño introduce parcialmente sus manos en el material (solamente la punta de los dedos).

6. El niño se sacude varias veces el material de sus manos o antebrazos.

7. Presenta respuesta de freezing.
8. Respuesta inicial es reiterada y no disminuye (no se habitúa).

9. Aumenta su nivel de alerta o de actividad, se desorganiza su conducta, se irrita emocionalmente o aumenta desatención.

Respuesta a pinceles y aire

1. Presenta respuesta cuando se le pasa pincel o cepillo.

2. Cuando se le pasa pincel o escobilla por primera vez, el niño detiene su conducta exploratoria y pregunta.

3. Cuando se le pasa pincel o escobilla las veces siguientes, el niño vuelve a detener su juego.

4. Presenta respuesta cuando se le sopla aire con una bombilla.

5. Cuando se le sopla con la bombilla por primera vez, el niño detiene su juego y pregunta.

6. Cuando se le sopla con la bombilla las veces siguientes, el niño vuelve a detener su juego.

7. Presenta respuesta de freezing.

\section{Observaciones Generales}

1. Niño baja sus mangas bruscamente en algún momento, o al finalizar alguno de los dos juegos.

Observaciones incidentales durante pruebas de Discriminación Táctil

1. Niño se rasca o frota la zona tocada.

2. Niño se golpetea la zona tocada.

Todos los ítems de la Pauta de Análisis provienen de un estudio piloto con 20 fichas clínicas de las que se extrajeron las expresiones verbales más frecuentes o cualitativamente significativas, y que se correlacionan con observaciones conductuales de dificultades en la modulación táctil reportadas en la literatura (Blanche, 2010; Bundy, 2002; Dunn, 2014; Lane, 2002; Miller \& Summers, 2001; Parham \& Mailloux, 2005; Royeen \& Lane, 1991; Royeen, 1986; Schoen, et al, 2008; Windsor, et al., 2001)

\section{Procedimiento de análisis}

Se diseñó un formulario en Microsoft Access ${ }^{\circledR}$, que contenía todos los ítems de la Pauta de Análisis, cruzando 
datos de cada individuo en relación con la fecha de la evaluación y de nacimiento. En éste se calificó la transcripción textual de las observaciones desde las fichas clínicas con la Pauta de Análisis. Posteriormente estos puntajes fueron calculados en Microsoft Excel ${ }^{\circledR}$, y luego se realizó el procesamiento estadístico de los datos con el software SPSS ${ }^{\circledR}$, realizándose estadística descriptiva análisis general exploratorio del comportamiento de las variables-, y pruebas no paramétricas de ambos grupos (debido a los ítems dicotómicos).

\section{Consideraciones éticas}

Como parte del procedimiento estándar de atención de los centros de atención terapéutica donde fue aplicado el instrumento de evaluación, los padres autorizaron expresamente la utilización de los resultados de las evaluaciones para investigación clínica, con la garantía de conservar el anonimato, en el consentimiento informado.

Por otra parte, la aplicación de un procedimiento de evaluación que no ha sido investigado previamente, se enmarca dentro del conjunto de observaciones clínicas de integración sensorial, que por medio del razonamiento clínico, sujeto a criterio y a normas (cuando éstas existen) contribuyen a una evaluación comprensiva de las dificultades ocupacionales del niño, así como muchos otros procedimientos naturalistas de evaluación de terapia ocupacional. Los padres fueron informados acerca de la versatilidad y alcance del procedimiento de evaluación general, y al finalizar la evaluación de su hijo, fueron informados de los resultados y conclusiones.

Finalmente, el procedimiento estructurado de observación clínica de la modulación táctil no presenta ningún riesgo de daño, y menos de manera permanente, sino sólo circunstancialmente un riesgo de incomodidad temporal para el niño, el cual se estima de fácil resolución en la mayoría de los casos, y en el marco de una relación terapéutica típica.

\section{Resultados}

\section{Aplicación de las pruebas realizadas}

Al ser un estudio retrospectivo, se tiene menos control de algunas variables, incluida la aplicación íntegra del protocolo. De las secciones propuestas en la Pauta de Análisis, el juego con espuma fue aplicado a un $94 \%$ de la muestra, el juego con sémola a un 80\%, la reacción a pinceles y aire a un $83 \%$ y test de discriminación a un $46 \%$, por lo cual este último será excluido del análisis (ítems 28 y 29). Igualmente, el ítem 27 ("niño baja sus mangas bruscamente en algún momento, o al finalizar alguno de los dos juegos") correspondiente a observaciones generales, sólo fue observado en una proporción muy baja de la muestra (12\%), por lo cual también se excluyó del análisis.

\section{Resultados por sección}

Se calcularon promedios de las respuestas a cada uno de los ítems a quienes se les aplicó el procedimiento, separados por grupos diagnósticos.

En el procedimiento completo, los 5 ítems cuya respuesta negativa pretende detectar a niños con bajo registro táctil $(1,2,11,20$ y 23), presentan una alta representatividad en ambos grupos (sobre $70 \%$ en el caso de los ítems 1, 11, 20 y 23), tal como era esperado, y aún mayor en niños DT.

Todos los ítems que hacían referencia a una respuesta de freezing para cada una de las secciones (9, 17 y 26) se observaron infrecuentes y homogéneos para ambos grupos (menor a 11\%), por lo cual se excluirán del análisis de cada tarea.

Específicamente, en el Juego con Espuma, se observa un mayor promedio en la respuesta de los niños DT con respecto a los niños NO DT, en todos los ítems, exceptuando el ítem 9 ya mencionado. Dos ítems ( 3 y 4) son reportados en más del 50\% de los niños DT, y menos del $25 \%$ en niños NO DT. Por otro lado, cinco ítems (5, $6,7,8$ y 10) son reportados en baja frecuencia en ambos grupos, sin embargo, dos de ellos (6 y 7) son particularmente infrecuentes en el grupo NO DT. (Ver gráfico en Figura 2). 
FIGURA 2 PROMEDIOS DE LAS RESPUESTAS A CADA ÍTEM EN JUEGO CON ESPUMA

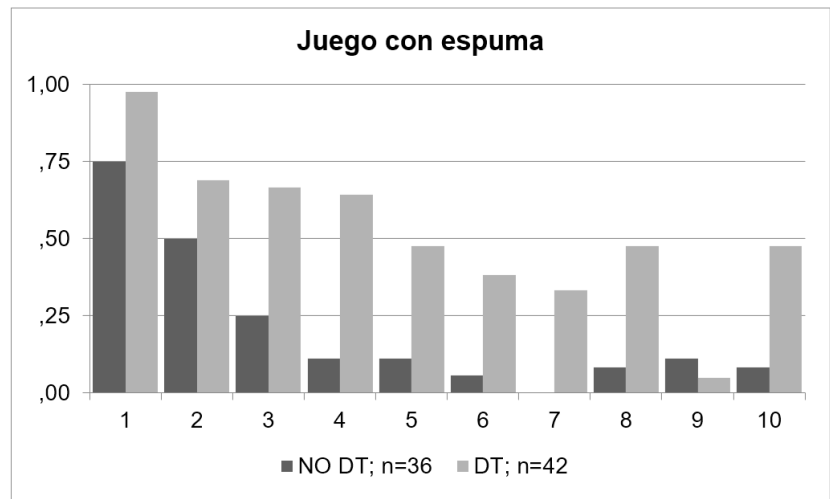

En el Juego con Sémola, también se observan promedios mayores en las respuestas de niños DT con respecto a los niños NO DT, en todos los ítems exceptuando el mencionado ítem 17. Dos ítems (12 y 16) están presentes en más del 50\% de los niños DT, y en menos del 33\% de los niños NO DT. Cinco ítems (13, 14,15, 18 y 19) son reportados en menos del 50\% de ambos grupos, pero particularmente en dos de ellos (19 y 15) se observa el contraste con una muy baja frecuencia en el grupo $\mathrm{NO}$ DT (menor al 7\%). (Ver gráfico en Figura 3).

\section{FIGURA 3 PROMEDIOS DE LAS RESPUESTAS A CADA ÍTEM} EN JUEGO CON SÉMOLA

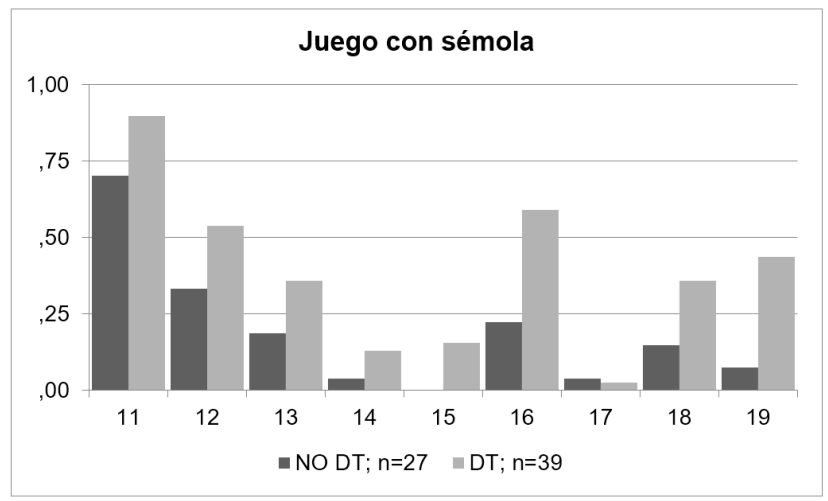

$\mathrm{Al}$ igual que todo lo anterior, los ítems referidos a la reacción a pinceles y aire, aplicados durante el Juego con Sémola, muestran una respuesta promedio mayor en niños DT que en niños NO DT. Dos ítems (21 y 24) se observan en más del 50\% de los niños DT y en menor proporción en niños NO DT. Los dos ítems restantes
(22 y 25) son menos frecuentes en ambos grupos, pero siempre en mayor frecuencia en niños DT.

\section{Pruebas no paramétricas}

En cada sección del procedimiento, se calcularon puntajes promedio de cada individuo para la sección completa. A continuación, se aplicó prueba no paramétrica a estos promedios para determinar la significación estadística de las diferencias observadas.

Se excluyeron de los promedios los ítems 9, 17, y 26 mencionados anteriormente, debido a la baja frecuencia de su observación para cualquiera de ambos grupos. Además, los ítems 1 y 2 en el Juego con Espuma, 11 en el Juego con Sémola, y 20 y 23 en Reacción a Pinceles y Aire, por su alta representación (y poca diferencia entre grupos), también serán omitidos de los promedios, ya que su función es, ante la ausencia de tales observaciones, detectar signos de hiporresponsividad.

Se reagruparon las secciones en dos conjuntos de observaciones para obtener los promedios: Juego con Espuma (ítems 3, 4, 5, 6, 7, 8 y 10), y Juego con Sémola -incluyendo la reacción a pinceles y aire- (ítems 12, 13, $14,15,16,18,19,21,22,24$ y 25 ).

La aplicación de la prueba no paramétrica U de MannWhitney de muestras independientes, arrojó una diferencia estadísticamente significativa entre ambos grupos diagnósticos tanto en el Juego con Espuma ( $p<$.o01), como en el Juego con Sémola ( $p<.001)$ (ver Tabla 2$)$. 
TABLA 2 ESTADÍSTICA DESCRIPTIVA Y PRUEBAS NO PARAMÉTRICAS (PRUEBA U DE MANN-WHITNEY DE MUESTRAS INDEPENDIENTES) PARA JUEGO CON ESPUMA Y JUEGO CON SÉMOLA, POR GRUPO DIAGNÓSTICO

\begin{tabular}{lllll}
\hline & & NO DT & DT & Nivel de significación \\
\hline \multirow{3}{*}{ Juego con Espuma } & Promedio & 0,10 & 0,49 & \\
& Desv. Est. & 0,20 & 0,35 & $\mathrm{p}<.001$ \\
& $\mathrm{~N}$ & 36 & 42 & \\
\hline \multirow{3}{*}{ Juego con Sémola } & Promedio & 0,15 & 0,41 & $\mathrm{p}<.001$ \\
& Desv. Est. & 0,22 & 0,29 & \\
& $\mathrm{~N}$ & 35 & 41 & \\
\hline
\end{tabular}

\section{Discusıón}

Hasta el momento, uno de los desafíos metodológicos en la evaluación y detección de dificultades de modulación táctil ha sido el obtener datos por medio de una medida del desempeño del niño, en lugar de sólo obtener información del reporte de los padres para hacer el diagnóstico. Las razones para no haber desarrollado estos instrumentos de evaluación pueden ser muy variadas y parecen estar relacionadas tanto con la estructura de una estrategia de evaluación que pueda gatillar estas respuestas defensivas, como con las variables a ser medidas.

Las respuestas defensivas suelen ser variables, muchas veces no son posibles de observar en contexto clínico, la falta de habituación parece ser un signo más relevante que la intensidad de la reacción (es decir, la respuesta acumulativa a los estímulos es más significativa que indicadores más obvios como una respuesta intensa y exagerada), existen factores sensoriales que pueden inhibir la respuesta táctil posterior (como la actividad física intensa -con sobreuso de propiocepción- previo a la exposición), y la manera en que los estímulos son presentados puede ser un factor más relevante que las cualidades propias de los mismos estímulos (es decir, estímulos que son aplicados pasivamente, o no visibles, en lugar de estar en control del individuo, anticipándose a qué esperar) (Ben-Sasson, et al., 2009; Blanche \& Reinoso, 2008; Blanche \& Schaaf, 2001; Blanche, 2010; Bundy, 2002; Mailloux \& Burke, 2008; Miller, et al., 2001; Parham \& Mailloux, 2005; Royeen \& Lane, 1991; Royeen, 1985; Windsor, et al., 2001). Además, se desconoce cuál sería el desempeño específico de las personas con defensividad táctil ante los estímulos que sean distinguibles del resto de la población.
Entonces, una razonable pregunta de investigación es: si se diseña un procedimiento de evaluación para la detección de la defensividad táctil, estructurado de tal forma que se controlen la mayor parte de las precauciones necesarias para gatillar una respuesta defensiva, ¿sería este procedimiento capaz de provocar estas respuestas, y distinguirlas del desempeño entre niños con y sin defensividad táctil? Este estudio presenta el desarrollo de un procedimiento estructurado de observación clínica de la modulación táctil, a través del desempeño del niño en dos juegos con exposición a materiales táctiles, analizado por medio de una pauta de análisis de estas respuestas, y comparando el desempeño de dos muestras clínicas de niños con y sin defensividad táctil.

En consecuencia, el resultado más relevante de este estudio es que el desempeño de niños con defensividad táctil sí difiere de manera estadísticamente significativa del desempeño de niños sin defensividad táctil en el procedimiento presentado, conclusión basada en la aplicación de pruebas no paramétricas (prueba $U$ de Mann-Whitney) al promedio de las puntuaciones tanto en el Juego con Espuma, como en el Juego con Sémola.

Refiriéndose específicamente a la estadística descriptiva de las pruebas, los promedios y medidas de dispersión obtenidos por cada grupo en ambas secciones del procedimiento, permiten deducir la cantidad de indicadores que pueden confirmar o desestimar el diagnóstico de defensividad táctil. Considerando que en el Juego con Espuma los promedios individuales obtenidos provienen de 7 ítems dicotómicos, se puede inferir que el desempeño de los niños DT se encuentra en un margen de 1 a 5 ítems aprobados (resultado obtenido a partir de una desviación estándar alrededor del promedio, multiplicado por 7 ítems). Haciendo el mismo ejercicio, el 
desempeño de niños NO DT fluctúa entre o y 2 ítems aprobados. Por lo tanto, respecto al Juego con Espuma, si un niño obtiene 3 o más ítems aprobados, tiene altas probabilidades de presentar defensividad táctil; al mismo tiempo, si un niño no presenta ninguno de los 7 ítems aprobados, tiene una alta probabilidad de no presentar defensividad táctil. Existe una superposición entre los grupos respecto a los individuos que presentan 1 o 2 ítems calificados positivamente, para los cuales otras medidas deben ser consideradas para confirmary rechazar un diagnóstico.

Igualmente, como los promedios del Juego con Sémola (incluyendo las reacciones a pinceles y aire) se obtienen de las respuestas a 11 ítems dicotómicos, se puede deducir que el desempeño de niños DT se encuentra de 2 a 7 ítems calificados afirmativamente, y el de niños NO DT está entre o y 4 ítems aprobados. De manera similar que en el Juego con Espuma, estos resultados se obtienen a partir de los valores de una desviación estándar sobre y bajo el promedio de cada grupo, multiplicado por 11 ítems. Entonces, la probabilidad de determinar el diagnóstico de defensividad táctil por medio del Juego con Sémola, es alto cuando el individuo obtiene 5 o más ítems aprobados, mientras que es más probable descartar esta determinación cuando aprueba sólo 1 o ningún ítem. En esta sección existe una mayor superposición entre los resultados de ambos grupos, sin poder determinarse la pertenencia a un grupo u otro cuando el individuo aprueba 2, 3 o 4 ítems.

Junto con responder la pregunta referida a cuántos signos conductuales se requieren para determinar el diagnóstico de defensividad táctil en ambas secciones del procedimiento, el análisis efectuado también puede responder cuáles signos conductuales son más relevantes para determinar el diagnóstico.

Para cada sección del procedimiento, ha sido relevante destacar cuáles ítems son los más frecuentes en niños DT, cuáles particularmente infrecuentes en niños NO DT, y en cuáles se observan grandes diferencias entre uno y otro grupo. Además, se ha podido analizar el contenido y el patrón de las respuestas, ordenándolas jerárquicamente, observando que ciertos ítems implican la calificación positiva de otros.

En el Juego con Espuma se propone el siguiente esquema orientativo de las respuestas (ver figura 4):

FIGURA 4. FLUJOGRAMA ORIENTATIVO DE LA CALIFICACIÓN DEL JUEGO CON ESPUMA SEGÚN PAUTA DE ANÁLISIS. LA DIRECCIÓN DE LAS FLECHAS INDICA QUE LA CALIFICACIÓN AFIRMATIVA DE LOS ÍTEMS SUPERIORES SUPONE LA MISMA CALIFICACIÓN EN LOS NIVELES INFERIORES. A LA INVERSA, LA CALIFICACIÓN NEGATIVA DE LOS NIVELES INFERIORES, IMPLICAN LA MISMA CALIFICACIÓN NEGATIVA EN LOS NIVELES SUPERIORES. LAS FLECHAS BLANCAS SUGIEREN LOS ÍTEMS A OBSERVAR EN PRIMER LUGAR, EN CASO QUE SEAN OBSERVADOS.

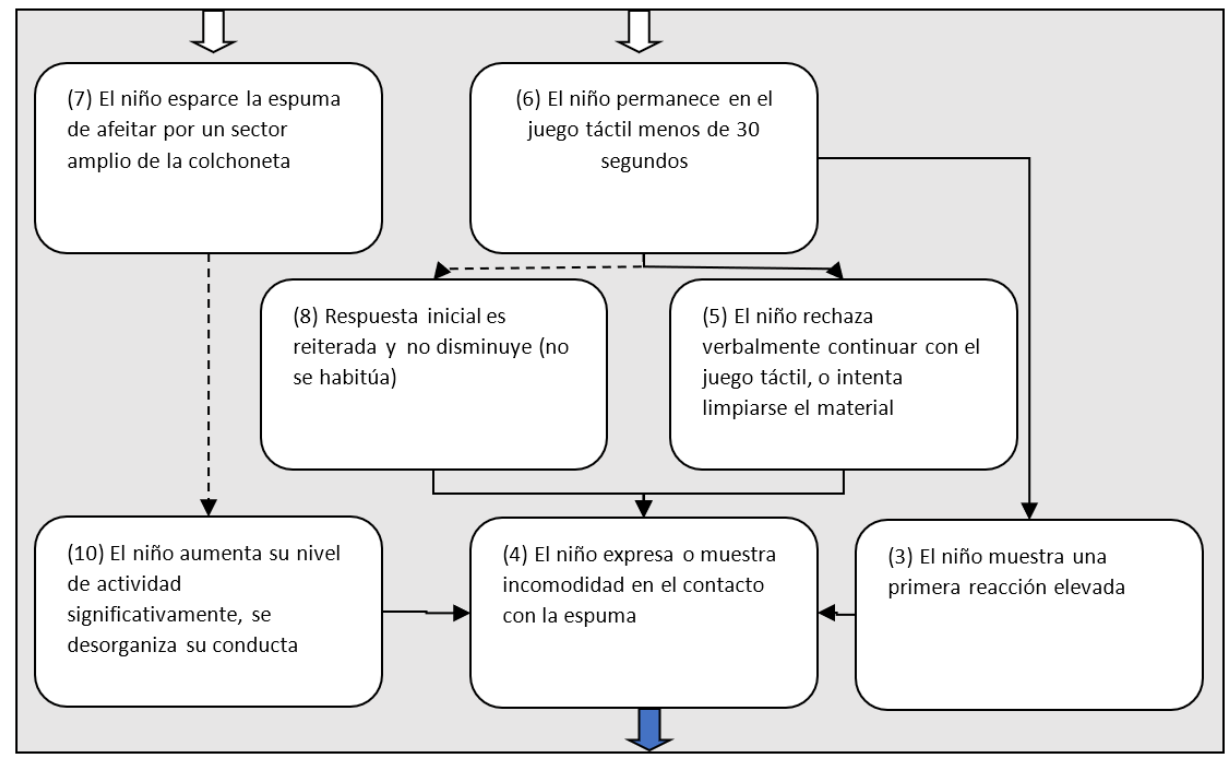


Los ítems más infrecuentes en el Juego con Espuma en el grupo NO DT son el ítem 6 ("Permanece en el juego táctil menos de 30 segundos") (6\%) y el ítem 7 ("El niño esparce la espuma de afeitar por un sector amplio de la colchoneta") (o\%). Además, de acuerdo con el flujograma, el ítem 6 supone la calificación afirmativa de los ítems 8, 5, 3 y 4, por lo cual, aun siendo no tan frecuente en niños DT (38\%), implica promedios más altos en la sección del procedimiento. Para comprobar esta hipótesis, a modo de ejercicio se tomaron los datos de la muestra considerando solamente a los individuos que calificaron positivamente en el ítem 6 , obteniendo en promedio 5,6 ítems aprobados (de hecho, ninguno obtuvo menos de 3 ítems), versus 1,6 ítems a quienes lo calificaron negativamente. Cabe mencionar que los indicadores más evidentes y explícitos (como abandonar el juego de manera abrupta) tienden a ser infrecuentes aún en niños con defensividad táctil, por lo que la mayoría de los signos que contribuirían con el diagnóstico son cualitativamente más sutiles, y la cantidad de éstos sería una medida relevante. Esto es similar a lo sugerido por Lane (2002) en relación con un patrón consistente en intensidad o cantidad de reacciones negativas al tacto.

El ítem 5 ("El niño rechaza verbalmente continuar con el juego táctil, o intenta limpiarse el material') se observa en una proporción 4 veces mayor en niños DT ( $48 \%$ frente a $11 \%$ ), y de acuerdo al esquema, atribuye respuesta afirmativa al ítem 4 subyacente. Los individuos calificados positivamente en el ítem 5, obtuvieron en promedio 5 ítems aprobados, mientras que los niños que los que no presentaron ese indicador, obtuvieron un promedio de o, 9 ítems.

Finalmente, el ítem 4 ("El niño expresa o muestra incomodidad en el contacto con la espuma"), se observa relativamente frecuente en niños DT (6o\%), e infrecuente en niños NO DT (11\%), y aunque su calificación positiva no supone más puntaje, el promedio obtenido por los individuos que lo calificaron negativamente es de o,48 ítems aprobados, lo cual aumenta la probabilidad de menor puntuación y podría sugerir descartar el diagnóstico.

Analizando el contenido de los ítems del Juego con Sémola, se puede apreciar que tienden a ser más homogéneos e independientes, por lo cual una jerarquización y un flujograma de éstos sería menos preciso que en la sección del Juego con Espuma. Sólo el ítem 14 (“El niño rechaza involucrarse o continuar en el juego con el material"), tiene una mayor probabilidad de obtener calificación en los demás ítems, pero, aunque éste es sumamente infrecuente en niños NO DT (4\%), es igualmente poco frecuente en niños DT (11\%). Esta observación, no obstante, permite deducir que la actividad del Juego con Sémola es agradable para la mayoría de los niños, permaneciendo involucrados en la actividad hasta finalizarla.

El ítem 16 ("El niño se sacude varias veces el material de sus manos o antebrazos") se estima como una observación relevante: es bastante frecuente en niños DT (59\%), y relativamente poco frecuente en niños NO DT $(22 \%)$, y además supondría la respuesta afirmativa al ítem 13 ("El niño manifiesta incomodidad verbal o gestualmente con el contacto"), ya que sacudirse el material implica incomodidad.

Respecto a los ítems durante la aplicación de pinceles y soplo de aire durante el Juego con Sémola, sólo se destacarían los ítems 21, 22 y 25 - "Cuando se le pasa pincel o escobilla por primera vez, el niño detiene su conducta exploratoria y pregunta", "Cuando se le pasa pincel o escobilla las veces siguientes, el niño vuelve a detener su juego" $y$ "Cuando se le sopla con la bombilla las veces siguientes, el niño vuelve a detener su juego", respectivamente- por presentar diferencias importantes entre ambos grupos (al menos $45 \%$ del grupo DT, y menos del $19 \%$ del grupo NO DT).

En síntesis, el procedimiento presentado puede generar ciertas respuestas defensivas que difieren significativamente entre niños con y sin defensividad táctil, y por lo tanto, permite contribuir con el proceso diagnóstico de la defensividad táctil. De esta manera, calificando positivamente en 3 o más ítems en el Juego con Espuma, y en 5 o más ítems en el Juego con Sémola es posible determinar el diagnóstico de defensividad táctil, mientras que éste se podría descartar cuando no se califica afirmativamente en ningún ítem del Juego con Espuma y hasta 1 ítem en el Juego con Sémola. También, es más probable establecer el diagnóstico cuando ciertos ítems específicos son calificados positivamente: ítem 6 ("Permanece en el juego táctil menos de 30 segundos"), ítem 5 ("El niño rechaza verbalmente continuar con el juego táctil, o intenta limpiarse el material"), ítem 14 ("EI niño rechaza involucrarse o continuar en el juego con el material"), o el ítem 16 ("El niño se sacude varias veces el material de sus manos o antebrazos"). 


\section{CONCLUSIÓN}

Se ha diseñado un procedimiento estructurado de observación clínica de la modulación táctil, siguiendo una serie de principios basados en la literatura, para gatillar respuestas defensivas a los estímulos táctiles en un contexto de juego, y que puedan ser observables por un evaluador. Este instrumento ha demostrado poseer un potencial discriminativo en el desempeño de niños con y sin defensividad táctil, logrando definir la cantidad de ítems necesarios y establecer cuáles ítems específicos aumentan la probabilidad de determinar el diagnóstico de defensividad táctil.

Junto con lo anterior, existen otros aspectos cualitativos interesantes de destacar: Por un lado, se trata de un procedimiento económico y de fácil implementación. Además, es un procedimiento atractivo para el niño, al estar basado en juego, por lo que tiende a mantenerse involucrado en la actividad, sin dañar el vínculo terapéutico, a pesar del potencial estrés por el contacto con estos materiales.

En el estudio presentado se identifican las siguientes limitaciones: Se trata de un estudio retrospectivo de fichas clínicas, elegidas por conveniencia, por lo cual no fue posible controlar todas las variables, como por ejemplo, la administración íntegra del procedimiento completo y la elección previa de algún instrumento de control. También, la muestra fue clínica tanto en el grupo control como en el grupo objetivo, existiendo una alta heterogeneidad en el grupo control y una muy baja representatividad de la población típica, y necesitándose una categorización dialéctica para la determinación de los grupos. Dicho de otro modo, el grupo control caracterizado como NO DT es una población que no presentaría defensividad táctil, pero sí presenta otros problemas de interés clínico (en salud mental o trastornos del desarrollo) y en su mayoría no presentan un desarrollo típico.

La muestra estudiada presenta una baja representación de mujeres, lo cual se debe a la mayor proporción de consultas de niños que de niñas. Además, el intervalo etario es muy amplio (desde los 2 años 6 meses hasta los 11 años), y no fue estratificado por grupos etarios, por lo que algunos tramos (especialmente sobre los 8 años), también se encuentran infrarrepresentados.
Como el estudio se realizó revisando fichas clínicas y calificando una pauta de análisis, en cuanto a la proyección en la aplicación clínica, se desconoce si la pauta puede ser utilizada directamente en la evaluación.

Y también respecto a la pauta de análisis, si bien la calificación con ítems dicotómicos presentó una ventaja inicial para cuantificar indicadores e identificar con mayor precisión cuáles de ellos son más relevantes, esto dificultó un procesamiento estadístico más enriquecedor, estableciendo correlaciones, o proyectar un análisis factorial para determinar subgrupos.

\section{Proyecciones}

La investigación presentada permite ampliar una línea de investigación referida a instrumentos de evaluación de la modulación táctil, y nuevas observaciones clínicas estructuradas.

En cuanto a la aplicación en la práctica clínica, este estudio logró caracterizar algunos procedimientos que corrientemente se han utilizado en terapia ocupacional (aunque de manera menos estructurada), pero de los que no se conocía su utilidad objetiva. De esta manera esta investigación ofrece algunos datos referidos a la cantidad de indicadores y a cuáles signos pueden sugerir defensividad táctil. Sin embargo, necesita ser investigado si la pauta de análisis puede ser utilizada directamente en la evaluación, o si necesita modificaciones para ese fin.

El procedimiento de observación clínica puede ser enriquecido con otros materiales y tareas que han sido utilizados de manera no estructurada en la tradición de la terapia ocupacional: actividades escolares con pegamento, cinta adhesiva, parches, masas y juguetes pegajosos (o como algunos de los utilizados por Lista y Blanche, 2020). Este eventual nuevo procedimiento debería seguir los principios y precauciones que guiaron teóricamente a este instrumento, y debería ser investigado con población típica.

Una nueva pauta de análisis debería ser diseñada simultáneamente con un eventual nuevo procedimiento de evaluación, transformándose en un protocolo de observación, y se sugiere modificar la escala de calificación por una con variables ordinales no dicotómicas. Un nuevo estudio debería ser dirigido, esta vez de manera 
prospectiva, validando el uso del instrumento en una población típica, y compararlo con el desempeño de niños con defensividad táctil, y con el de niños con diagnósticos con conocida comorbilidad (por ejemplo: Síndrome de X Frágil, Trastorno por Déficit Atencional, Trastornos del Espectro Autista). Es requerido aumentar la representación de niñas en la muestra y estratificar por rangos etarios con rigurosidad. Una de las posibles preguntas de investigación puede ser si existe una tendencia a expresarse en menor medida las reacciones defensivas conforme avanza la edad, en virtud de la maduración de los procesos inhibitorios del sistema nervioso central (Davies, Chang \& Gavin, 2009), de manera que se puedan describir trayectorias del desarrollo de las habilidades de modulación, y definir la utilidad de estos instrumentos en individuos de mayor edad.

Este estudio fue financiado con recursos propios del investigador y correspondió a su tesis de Magíster en Estrategias de Intervención en Salud Mental Infantil. La Universidad del Desarrollo proporcionó guía teórica y metodológica por medio del profesor Francisco Ceric, $\mathrm{PhD}$.

\section{ReferenCiAs BibliográfiCAS}

Ayres, A. J. (1980). Southern California Sensory Integration Tests: Manual (rev ed.). Los Angeles: Western Psychological Services.

Bates, J., Bennett Freeland, C. A., \& Lounsbury, M. (1979). Measurement of Infant Difficultness. Child Development, 50(3), 794-803.

Ben-Sasson, A., Carter, A. S., \& Briggs-Gowan, M. J. (2009). Sensory over-responsivity in elementary school: Prevalence and socialemotional correlates. Journal of Abnormal Child Psychology, 37, 705-716.

Blanche, E. I. (2010). Observaciones basadas en la teoría de la integración sensorial. Torrance, CA: Pediatric Therapy Network.

Blanche, E. I., \& Reinoso, G. (2008). The use of clinical observations to evaluate proprioceptive and vestibular functions. OT Practice, 13(17), CE1-CE6.

Blanche, E. I., \& Schaaf, R. C. (2001). Proprioception: A cornerstone of sensory integration intervention. In S. S. Roley, E. I. Blanche, \& R. C. Schaaf (Eds.), Understanding the nature of sensory integration with diverse populations (pp. 109-124). Austin, TX: Pro-Ed.

Blanche, E. I., Bodison, S., Chang, M. C., \& Reinoso, G. (2012). Development of the Comprehensive Observations of Proprioception (COP): Validity, reliability, and factor analysis. American Journal of Occupational Therapy, 66, 691-698.
Blanche, E. I., Reinoso, G., \& Blanche Kiefer, D. (2014). Observaciones Clínicas Sensorio-Motoras. Los Angeles, CA: SensoryMetrics.

Blanche, E. I., Reinoso, G., \& Blanche Kiefer, D. (2020). Using Clinical Observations within the Evaluation Process. In A. C. Bundy, \& S. J. Lane (Eds.), Sensory integration: Theory and practice (3nd ed., pp. 222-242). Philadelphia: F. A. Davis. (2014).

Bundy, A. C. (2002). Assessing Sensory Integrative Dysfunction. In A. C. Bundy, S. J. Lane, \& E. A. Murray (Eds.), Sensory Integration: Theory and practice (2nd ed., pp. 169-198). Philadelphia: F. A. Davis.

Bundy, A. C. (2020). Assessing Sensory Intagrative Dysfunction without the SIPT. In A. C. Bundy, \& S. J. Lane (Eds.), Sensory integration: Theory and practice (3nd ed., pp. 243-255). Philadelphia: F. A. Davis.

Bundy, A. C., \& Lane, S. J. (2020). Sensory integration: A. Jean Ayres's theory revisited. In A. C. Bundy, \& S. J. Lane (Eds.), Sensory integration: Theory and practice (3nd ed., pp. 2-20). Philadelphia: F. A. Davis.

Bundy, A. C., \& Murray, E. A. (2002). Sensory integration: A. Jean Ayres's theory revisited. In A. C. Bundy, S. J. Lane, \& E. A. Murray (Eds.), Sensory integration: Theory and practice (2nd ed., pp. 3-33). Philadelphia: F. A. Davis.

Calderón, D. (2016). Desarrollo de un procedimiento estructurado de observación clínica de la defensividad tactil (Tesis de Magister). Universidad del Desarrollo, Santiago, Chile. Recuperado de http://hdl.handle.net/11447/2010

Davies, P. L., Chang, W.-P., \& Gavin, W. J. (2009). Maturation of sensory gating performance in children with and without sensory processing disorders. International Journal of Psychophysiology, 72, 187-197.

Dunn, W. (2014). Sensory Profile 2 User's Manual. San Antonio, TX: Pearson. Dunn, W., Little, L., Dean, E., Robertson, S., \& Evans, B. (2016). The State of the Science on Sensory Factors and Their Impact on Daily Life for Children: A Scoping Review. OTJR: Occupation, Participation and Health, 36(2S), 3S-26S.

Eeles, A. L., Spittle, A. J., Anderson, P. J., Brown, N., Lee, K. J., Boyd, R. N., \& Doyle, L. W. (2013). Assessments of sensory processing in infants: a systematic review. Developmental Medicine \& Child Neurology, 55, 314-326.

Lane, S. J. (2002). Sensory Modulation. In A. C. Bundy, S. J. Lane, \& E. A. Murray (Eds.), Sensory integration: Theory and practice (2nd ed., pp. 101-122). Philadelphia: F. A. Davis.

Lane, S. J. (2020). Sensory Modulation Functions and Disorders. In A. C. Bundy, \& S. J. Lane (Eds.), Sensory integration: Theory and practice (3rd ed., pp. 151-180). Philadelphia: F. A. Davis.

Lista, C. y Blanche, E. I. (2020). Diferencias en el procesamiento táctil en niños con diagnóstico de autismo y niños típicos entre 4.0 años y 5.11 años. Validez de una medida de evaluación del procesamiento táctil. Revista Argentina de Terapia Ocupacional, 6(1), 45-49

Mailloux, Z., \& Burke, J. P. (2008). Play and the Sensory Integrative Approach. In L. D. Parham, \& L. S. Fazio (Eds.), Play in Occupa- 
tional Therapy for Children (2nd ed., pp. 263-278). St. Louis, MO: Mosby Elsevier.

Mailloux, Z., Mulligan, S., Roley, S. S., Blanche, E., Cermak, S., Coleman, G. G., .. . Lane, C. J. (2011). Verification and Clarification of Patterns of Sensory Integrative Dysfunction. American Journal of Occupational Therapy, 65, 143-151.

Miller, L. J., \& Summers, C. (2001). Clinical Applications in Sensory Modulation Dysfunction. In S. Roley, E. Blanche, \& R. Schaaf (Eds.), Understanding the nature of sensory integration with diverse populations (pp. 247-274). Austin, TX: Pro-Ed.

Miller, L. J., Anzalone, M. E., Lane, S. J., Cermak, S. A., \& Osten, E. T. (2007). Concept evolution in sensory integration: A proposed nosology for diagnosis. American Journal of Occupational Therapy, 61, 135-140.

Miller, L. J., Coll, J. R., \& Schoen, S. A. (2007). A randomized controlled pilot study of the effectiveness of occupational therapy for children with sensory modulation disorder. American Journal of Occupational Therapy, 61, 228-238.

Miller, L. J., Reisman, J. E., McIntosh, D. N., \& Simon, J. (2001). An Ecological Model of Sensory Modulation. In S. Roley, E. Blanche, \& R. Schaaf (Eds.), Understanding the nature of sensory integration with diverse populations (pp. 57-59). Austin, TX: Pro-Ed.

Mulligan, S. (2020). Assessment of sensory integration functions using the Sensory Integration and Praxis Tests. In A. C. Bundy, \& S. J. Lane (Eds.), Sensory integration: Theory and practice (3nd ed., pp. 208-221). Philadelphia: F. A. Davis.

Mulligan, S., Schoen, S. A., Miller, L. J., Valdez, A., \& Magalhaes, D. (2019). The Sensory Processing 3-Dimensions Scale: Initial Studies of Reliability and Item Analyses. The Open Journal of Occupational Therapy, 7(1). https://doi.org/10.15453/2168-6408.1505

Parham, L. D., \& Mailloux, Z. (2005). Sensory Integration. In J. Case-Smith (Ed.), Occupational Therapy for Children (5th ed., pp. 356-411). St. Louis: Elsevier Mosby.

Rothbart, M. K., Ahadi, S. A., Hershey, K. L., \& Fisher, P. (2001). Investigations of Temperament at Three to Seven Years: The Children's Behavior Questionnaire. Child Development, 72, 1394-1408.

Royeen, C. B. (1985). Domain Specifications of the Construct Tactile Defensiveness. American Journal of Occupational Therapy, 39(9), 596-599.

Royeen, C. B. (1986). The Development of a Touch Scale for Measuring Tactile Defensiveness in Children. American Journal of Occupational Therapy, 40(6), 414-419.

Royeen, C. B., \& Lane, S. J. (1991). Tactile processing and sensory defensiveness. In A. Fischer, E. Murray, \& A. Bundy, Sensory integration: Theory and practice (pp. 108-133). Philadelphia: F. A. Davis.

Schoen, S. A., Miller, L. J., \& Green, K. E. (2008). Pilot study of the Sensory Over-Responsivity Scales: Assessment and inventory. American Journal of Occupational Therapy, 62, 393-406.
Schoen, S. A., Miller, L. J., \& Sullivan, J. C. (2014). Measurement in sensory modulation: The Sensory Processing Scale Assessment. American Journal of Occupational Therapy, 68, 522-530.

Windsor, M. M., Roley, S. S., \& Szklut, S. (2001). Assessment of sensory integration and praxis. In S. S. Roley, E. I. Blanche, \& R. C. Schaaf (Eds.), Understanding the nature of sensory integration with diverse populations (pp. 215-245). Austin, TX: Pro-Ed.

\section{Anexo}

Protocolo de Observación Clínica de la Modulación Táctil, Pauta de Análisis, y Definiciones Operacionales de los Ítem, disponibles en https://drive.google. com/file/d/1XOxnA3y2YIBkCk7XggZhZRust1lgCV36/ view? usp=sharing 
